TITLE:

\title{
Discrepancy between explicit judgement of agency and implicit feeling of agency: Implications for sense of agency and its disorders.
}

\section{$\operatorname{AUTHOR}(\mathrm{S}):$}

Saito, Naho; Takahata, Keisuke; Murai, Toshiya;

Takahashi, Hidehiko

\section{CITATION:}

Saito, Naho ...[et al]. Discrepancy between explicit judgement of agency and implicit feeling of agency: Implications for sense of agency and its disorders.. Consciousness and cognition 2015, 37: 1-7

\section{ISSUE DATE:}

2015-12

URL:

http://hdl.handle.net/2433/201984

\section{RIGHT:}

(c) 2015. This manuscript version is made available under the CC-BY-NC-ND 4.0 license

http://creativecommons.org/licenses/by-nc-nd/4.0/; The full-text file will be made open to the public on 6 August 2017 in accordance with publisher's 'Terms and Conditions for Self-Archiving'.; This is not the published version. Please cite only the published version.; この論文は出版社版でありません。引用の際には出版社版をご確認ご利用ください。 
1 Title: Discrepancy between explicit judgement of agency and implicit feeling of

2 agency: implications for sense of agency and its disorders

3

4 Author names: Naho Saito ${ }^{\text {a)}}$, Keisuke Takahata ${ }^{\text {b) }}$, Toshiya Murai ${ }^{\text {a) }}$, Hidehiko

5 Takahashi $^{\text {a) }}$

6

7 Author affiliations:

8 a) Department of Psychiatry, Graduate School of Medicine, Kyoto University, 54

9 Shogoin-Kawahara-cho, Sakyo-ku, Kyoto 606-8507, Japan

10 b) Molecular Imaging Center, National Institute of Radiological Sciences, 4-9-1

11 Anagawa, Inage-ku, Chiba 263-8555, Japan

12

13 Corresponding author: Hidehiko Takahashi, M.D., Ph.D.

14 Department of Psychiatry, Kyoto University Graduate School of Medicine,

1554 Shogoin-Kawahara-cho, Sakyo-ku, Kyoto 606-8507, Japan

16 E-mail: hidehiko@kuhp.kyoto-u.ac.jp

17 


\section{Abstract}

21 The sense of agency refers to the feeling of authorship that " $\mathrm{I}$ am the one who is 22 controlling external events through my own action”. A distinction between explicit 23 judgement of agency and implicit feeling of agency has been proposed theoretically. 24 However, there has not been sufficient experimental evidence to support this distinction. 25 We have assessed separate explicit and implicit agency measures in the same population 26 and investigated their relationships. Intentional binding task was employed as an 27 implicit measure and self-other attribution task as an explicit measure, which are known 28 to reflect clinical symptoms of disorders in the sense of agency. The results of the 29 implicit measure and explicit measure were not correlated, suggesting dissociation of the explicit judgement of agency and the implicit feeling of agency.

\section{Key words}

sense of agency; voluntary action; feeling of agency; judgement of agency; central monitoring; intentional binding

\section{Introduction}

The sense of agency refers to the feeling of authorship that "I am the one who is controlling external events through my own action”. This sense is a central component of self-awareness (Gallagher, 2000), and its underlying neural mechanisms have been reported (David, Newen, \& Vogeley, 2008). Symptoms of psychiatric and neurological diseases can be explained as a disruption of the sense of agency; examples of such are schizophrenia, conversion disorder, anarchic hand syndrome, and anosognosia for one's own hemiparesis (Kranick et al., 2013; Synofzik, Vosgerau, \& Newen, 2008b). For example, delusion of control in schizophrenia is a passivity experience that "My action is being controlled by others", which is an alteration in the sense of agency. These symptoms teach us that the sense of agency, a fallible process (Blakemore, Wolpert, \& Frith, 2002), requires reliable and objective clinical indicators. Measures of agency have been invented and assessed to give a fundamental understanding of self-awareness

49 (Haggard, Clark, \& Kalogeras, 2002; Nielsen, 1963). At the same time, these measures

50 have served as objective indicators to assess the subjective symptoms of the diseases 51 (Daprati et al., 1997; Franck et al., 2001; Haggard, Martin, Taylor-Clarke, Jeannerod, \& 
Franck, 2003; Kranick et al., 2013; Maeda et al., 2013; Wolpe et al., 2014).

There have been two distinct ways in measuring the sense of agency - explicit and implicit. Explicit measures address the sense of agency by obtaining a direct report of how they attribute the effect of their action. In a pioneering experiment, participants were asked to draw a line on a piece of paper, and at the same time the experimenter gave manual visual feedback that was in concordance with or in discordance with their actual movements (Nielsen, 1963). This paradigm has been modified in various works to test the participant's ability to distinguish the actions they have performed and the actions performed by others (Daprati et al., 1997; Farrer et al., 2008; Franck et al., 2001; Maeda et al., 2012). In the study by Franck and colleagues (Franck et al., 2001), participants were given visual feedback of a voluntary action as a virtual hand, which moved in concordance with or in discordance with their movements. They were asked later on if the feedback corresponded with their actual movement or not. Patients with delusion of control in schizophrenia gave more "yes" answers to this question than normal participants did, indicating a correlation of clinical passivity experiences with the experimental attribution of actions.

However, it has been pointed out that explicit measures of agency can be subject to response bias (Wegner, 2003), and the need for indirect markers of agency has been discussed. The "intentional binding” effect focusing on temporal attraction between the perceived time of actions and their effects is a widely used quantitative method (Ebert \& Wegner, 2010). Participants perform a volitional button press at the timing of their own choosing. They judge the timing of their volitional button press on the basis of Libet's clock method (Libet, Gleason, Wright, \& Pearl, 1983). The button press will be followed by an auditory tone 250ms later. This is considered the effect of the action. They also judge the timing of the tone. A compression of timing judgments in action and its effect (the "intentional binding" effect) is known in the case of volitional actions but not in the case of non-volitional actions, and thus this method has been regarded as an implicit way to measure the sense of agency (Ebert \& Wegner, 2010). The intentional binding effect has also been observed to change in accordance with the passivity experiences in diseases (Haggard et al., 2003; Kranick et al., 2013; Wolpe et al., 2014), which can serve as a quantitative indicator.

So far, a two-step distinction in the formation of implicit and explicit sense agency has been proposed (Synofzik, Vosgerau, \& Newen, 2008a; Synofzik, Vosgerau, \& Voss, 
2013), complementary to the central monitoring theory (i.e. “comparator model”) (C. D. Frith, Blakemore, \& Wolpert, 2000). In the central monitoring theory, the sensory consequence of our action is predicted based on internal signals such as efference copy of the motor command. Comparison of the prediction with sensory afference will enable us to distinguish self-produced sensory information from externally caused events. Congruency of the predicted with sensory afference will lead to an interpretation that the action has been caused by our self, while incongruency will lead to an interpretation that the action has been caused externally. The sense of agency is explained in the final stage of action execution by a single mechanism in this framework. Recent studies pointed out that the sense of agency is not only based on internal signals but also modulated by various context cues (Moore \& Haggard, 2008; Moore, Wegner, \& Haggard, 2009; Takahata et al., 2012; Voss et al., 2010; Wegner, 2003). These observations have led to arguments that the sense of agency holds a more complex structure, with multiple levels involving different processes (Fletcher \& Frith, 2009; C. Frith, 2012; Moore \& Fletcher, 2012; Synofzik et al., 2008a; Synofzik et al., 2013). The presence of problematic cases of the central monitoring theory in explaining the sense of agency both in healthy subjects and in patients with passivity experiences has also been pointed out (Synofzik et al., 2008a). Accordingly, a two-step distinction is proposed between the level of the "feeling of agency" and the "judgement of agency" (Synofzik et al., 2008a). The first-level feeling of agency is the non-conceptual, low-level feeling of being an agent. It refers to the implicit aspect of agency, which is closely related to action regulation or perceptual processing. The second-level judgement of agency is the conceptual, interpretative judgement of being an agent of an action. It refers to the explicit judgement of self-other attribution, which is closely related to background beliefs or context cues (Synofzik et al., 2008a). However, few experimental studies have approached the relationship between these two aspects of the sense of agency (Barlas \& Obhi, 2014; Dewey \& Knoblich, 2014; Ebert \& Wegner, 2010; Moore, Middleton, Haggard, \& Fletcher, 2012).

Recently, some efforts have been made to investigate both explicit and implicit measures of agency in a single task (Ebert \& Wegner, 2010). However, the majority of previous experimental studies of psychiatric and neurological diseases assessed either explicit or implicit measures of agency (David et al., 2008), and they reported mixed results (e.g. exaggerated or decreased sense of agency in schizophrenia) (Voss et al 
118 2010, Maeda et al 2013). Comparison of the traditional tasks that have frequently been

119 used for clinical cases will facilitate the interpretation of the results of clinical studies

120 from the perspective of the structures of the tasks. Thus, we separately assessed both

121 explicit and implicit agency measures in the same population and investigated their 122 relationships.

\section{2. Materials and Methods}

\section{2.1. Participants}

126 Twenty-five subjects (thirteen female, mean age $=64.9$ years, $\mathrm{SD}=2.9$ years)

127 participated in the study. Participants with known neurological or psychiatric history

128 were excluded from the study. All the participants were right-handed according to the

129 Edinburg Inventory (Oldfield, 1971). Participants underwent two experiments. The

130 implicit task was conducted first and the explicit task next, in order to keep the

131 participants naïve to the study purpose. Written informed consent was obtained from

132 each participant. Participants were paid for their participation. This study was approved

133 by the ethics committee of Kyoto University Graduate School and Faculty of Medicine.

134

\section{2.2. Procedures and analysis}

136 2.2.1. Experiment 1- Implicit task

\section{2.2.1.1. Procedures}

138 The sequence of events from a previous study (Haggard et al., 2002), known as 139 intentional binding task, was employed. The task consisted of four conditions: (1) 140 agency action, (2) agency tone, (3) baseline action and (4) baseline tone. In each 141 condition, a blank screen was first presented, followed by a picture of a clock face and 142 clock hand. The clock-hand was $12 \mathrm{~mm}$ long, which rotated clockwise for a full rotation 143 in $2560 \mathrm{~ms}$. The clock face was marked with 12 conventional interval positions (5,10,15, 144 etc.). Initial positions of the clock-hand were chosen randomly from the 12 positions of 145 the clock. The clock-hand remained stationary at the initial position for $500 \mathrm{~ms}$, and 146 then began to rotate. Procedures during the clock-hand rotation were as follows. In the 147 agency action and agency tone conditions, participants performed a voluntary action. 148 Participants performed a key press at a time of their own choosing during the 149 clock-hand rotation. They were instructed to avoid responding at a pre-decided clock 150 position, or during the first half-rotation of the clock hand. Each key press triggered a 
151 tone after a fixed period of $250 \mathrm{~ms}$. In the agency action condition, participants were

152 asked to report the perceived onset time of their voluntary key press as judged by the

153 perceived position of the clock hand. Similarly in the agency tone condition,

154 participants were asked to report the perceived onset time of the triggered tone. In the

155 baseline action condition, participants performed a voluntary key press at the time of

156 their own choosing, but it did not yield a tone. Participants reported the perceived onset

157 time of the voluntary key press. In the baseline tone condition, participants did not press

158 a key but instead waited for a tone to be delivered, judging the onset time at which they

159 heard the tone. Before running the experiment, participants performed a practice session.

160 Each category of conditions was tested in separate blocks, in pseudo-randomized order

161 consisting of 24 trials. Missed trials were repeated. After completing the task with one

162 hand, participants conducted the task with the other hand. The order of right and left

163 hand was counterbalanced across participants. All stimuli were displayed using

164 Superlab 4.5 software.

165

166

\subsubsection{Data analysis}

167 For the implicit task (experiment 1), the perceived time of action or tone in each trial 168 was compared with the actual onset time, and a mean temporal estimation was 169 calculated for each block. The mean estimation for actions and tones in the baseline 170 condition was subtracted from that in the agency condition. Subtracting these baseline 171 estimates allowed us to calculate the shift in the perceived time of the tone when caused 172 by the action. These shifts served as measures of action binding and tone binding,

173 respectively. These subtracted measures correspond to the perceived linkage between 174 action and effect, and larger values indicate stronger perceived linkage. Finally, overall

175 binding was defined as action binding minus tone binding. The bindings of the two 176 hands were compared by paired t-tests.

\subsubsection{Experiment 2- Explicit task}

179 2.2.2.1. Procedures

180 A simplified task from a previous study (Franck et al., 2001) was employed. 181 Participants were asked to hold a joystick that was connected to a computer. A black 182 cover covered the joystick so that the participants could not see their actual movement. 183 Instead, an image of an electronically constructed virtual hand was presented to the 
184 participants on a computer screen as a feedback during the procedure. Participants were 185 instructed that "their hand" would appear on the computer screen. A specially designed 186 program synthesized images of a virtual hand holding a joystick and the virtual hand 187 moved according to the position that was actually held by the participants. The 188 movement of the joystick was presented dynamically on the screen with an intrinsic 189 delay of $16 \mathrm{~ms}$.

190 In each trial, an image of a virtual hand was presented for 10 seconds after a blank 191 screen, during which time participants were asked to move the joystick according to 192 their own choosing. The movement could be executed in four directions (right, left, 193 back, and forth). Immediately after the virtual hand disappeared, participants were 194 asked a yes-or-no question as follows: "Did the movement you saw on the screen 195 correspond to the movement you made with your hand?”

196 The task consisted of three categories of conditions: (1) neutral, (2) with angular biases, 197 and (3) with temporal biases. In the neutral condition, the virtual hand moved exactly 198 according to the movements the participants made with the joystick. In the angular 199 biases condition, a given angular value $\left(5^{\circ}, 10^{\circ}, 15^{\circ}\right.$, and $\left.20^{\circ}\right)$ was introduced as a gap 200 between the movements of the virtual hand and the joystick. In the temporal biases 201 condition, a given time delay (50, 100, 150, 200, 300, 400, and $500 \mathrm{~ms}$ ) was introduced 202 as a gap between the movements of the virtual hand and the joystick.

203 Trials with angular biases and trials with temporal biases were run four times for each 204 type of gap. Neutral trials were run 12 times. The order of presentation of all trials was 205 randomized for each subject. Before running the experiment, participants performed a 206 practice session. Missed trials were repeated. After completing the task with one hand, 207 participants conducted the task with the other hand. The order of right and left hand was 208 counterbalanced across participants.

209

\subsubsection{Data analysis}

211 For the explicit task (experiment 2), there could potentially be two types of errors: "yes" 212 responses for trials with a bias, and "no" responses for neutral trials. For data analysis, 213 “yes” responses were focused upon, reflecting the participants' ability to recognize the 214 movement as their own. "Yes" responses of the two hands were examined by repeated 215 measures ANOVA with event (each bias) and hand (right versus left), for angular and 216 temporal gaps separately. The data were converted into a 0-1 estimate ( 0 for "no" and 1 
217 for “yes” responses), to fit into a logistic regression model of $\mathrm{Y}=1 /(1+\exp (-(\mathrm{a}+\mathrm{bX})))$.

218 The slope coefficient (b) was calculated for each subject, as these slopes provide

219 estimates about how strictly a subject would draw an explicit judgement of agency. The

$22050 \%$ threshold $(-\mathrm{a} / \mathrm{b})$ for the total data was also calculated.

221 Lastly, correlations between the results of the implicit task and the explicit task were 222 explored by Spearman's rank correlation analysis. A p-value of less than 0.05 was 223 considered significant in all analyses.

224

225

\section{Results}

226

\subsection{Implicit task}

227 The perceived time of actions of the baseline condition was -176.5 (SD: 106.8) ms in 228 the right-hand trials, and -187.0 (SD: 96.0) ms in the left-hand trials. The perceived time 229 of tones in the baseline condition was -50.6 (SD: 61.5 ) ms. There was a positive shift in 230 the perceived time of actions in the agency condition compared to the baseline condition 231 (action binding) [right: 64.2 (SD: 119.4) ms, $p=0.013$; left: 78.3 (SD: 117.7) ms, $232 p=0.003]$. At the same time, there was a negative shift in the perceived time of tones in 233 the agency condition compared to baseline condition (tone binding) [right: -113.1 (SD: 234 155.5) ms, $p=0.001$; left: -114.5 (SD: 171.3) ms, $p=0.003$ ]. These results indicate that 235 actions were perceived later when they were followed by tones, and tones produced by 236 voluntary actions were perceived earlier than baseline tones. Overall binding was 237 calculated as action binding minus tone binding [right: 177.3 (SD: 218.3) ms; left: 192.8 238 (SD: 214.1) ms].

239 Action binding, tone binding and overall binding between the right and left hand were 240 highly correlated [action binding: $r=0.877, p=0.000$; tone binding: $r=0.902, p=0.000$; 241 overall binding: $r=0.908, p=0.000]$, and did not show significant difference in paired 242 t-tests [action: $t(24)=1.195, p=0.244$; tone: $t(24)=0.093, p=0.927$; overall: $t(24)=0.762$, $243 p=0.453$ ] (Figure 1). The averaged data of the right and left hand for each participant 244 were focused in the following correlation analyses. The averaged action binding was $24571.2(\mathrm{SD}=114.9) \mathrm{ms}$, tone binding was $-113.8(\mathrm{SD}=159.3) \mathrm{ms}$, and overall binding 246 was $185.1(\mathrm{SD}=224.4) \mathrm{ms}$.

\subsection{Explicit task}

249 Repeated measures ANOVA with angular bias $\left(0^{\circ}, 5^{\circ}, 10^{\circ}, 15^{\circ}\right.$, and $\left.20^{\circ}\right)$ and hand 
250 (right and left) revealed a main effect of angular bias $(F(2.9,70.8)=72,17, p=0.000)$, no 251 angular bias $\times$ hand interaction $(F(1.7,41.9)=1.47, p=0.24)$, and no main effect of hand $252(F(1,24)=1.37, p=0.25)$. Similarly, repeated measures ANOVA with temporal bias $(0,50$, $253100,150,200,300,400$, and $500 \mathrm{~ms}$ ) and hand (right and left) revealed a main effect of 254 temporal bias $(F(3.2,76.8)=92.60, p=0.000)$, no temporal bias $\times$ hand interaction $255(F(4.3,103.0)=1.19, p=0.319)$, and no main effect of hand $(F(1,24)=1.34, p=0.259)$.

256 These results indicate that the participants' attribution of the movement was affected by 257 angular biases and by temporal biases, but not by their handedness (Figure 2).

258 Next, the data as a 0-1 estimate (0 for "no" and 1 for "yes" responses) were fit into a 259 logistic regression model of $\mathrm{Y}=1 /(1+\exp (-(\mathrm{a}+\mathrm{bX})))$. The data of the right and left hand 260 were included together in the following analysis. The slope coefficient (b) was 261 calculated for each subject. The average slope coefficient (b) for each participant was $-1.10(\mathrm{SD}=1.75)$ for angular biases condition, and $-0.022(\mathrm{SD}=0.014)$ for temporal biases condition. The $50 \%$ threshold $(-\mathrm{a} / \mathrm{b})$ for the total data was revealed to be $9.5^{\circ}$ for the angular biases condition and $170.9 \mathrm{~ms}$ for the temporal biases condition. For this reason, “yes" responses in the $5^{\circ}$ and $10^{\circ}$ for the angular biases, and $150 \mathrm{~ms}$ and $200 \mathrm{~ms}$ for the temporal biases were focused upon in the following correlation analyses. The average percentage of “yes” responses was 75.5\% (SD $=27.4)$ for $5^{\circ}$ angular bias, $46.0 \%(\mathrm{SD}=35.1)$ for $10^{\circ}$ angular bias, $52.0 \%(\mathrm{SD}=35.8)$ for $150 \mathrm{~ms}$ temporal bias, and $38.0 \%(S D=31.3)$ for $200 \mathrm{~ms}$ temporal bias.

\subsection{Relationship between implicit and explicit task}

272 The measures in the implicit task (action binding, tone binding and overall binding) were compared with each of the slope coefficients (b) in the explicit task. Then the measures in the implicit task were also compared with the numbers of "yes" responses around the 50\% threshold in the explicit task. There was no significant correlation between bindings in the implicit task and the measures in the explicit task (Table 1).

\section{Discussion}

279 In this study we have assessed two distinct methods of measuring the sense of agency 280 and investigated their relationships. We employed methods that are widely recognized 281 as being in accordance with clinical symptoms of the disorders in the sense of agency: 282 “intentional binding” task as an implicit measure and self-other attribution task as an 
283 explicit measure. We found a discrepancy between implicit intentional binding and 284 explicit self-other attribution.

285 In the intentional binding task (experiment 1), participants experienced actions as 286 shifted towards their subsequent effects, while effects were perceived as shifted towards 287 the preceding action. This was compatible with previous findings and can be regarded 288 as a bias to intensify the causal relationship between action and its consequence 289 (Haggard et al., 2002). In the explicit self-other attribution task (experiment 2), 290 participants gave most attribution of the feedback to themselves when the movement 291 had not deviated from their actual movement, and this tendency decreased as the 292 angular bias and temporal bias became more obvious. At the same time, this means that 293 the distorted sensory feedbacks could be attributed to their own movement even in cases 294 of certain discrepancies, with continuous recalibration. This observation does not 295 strictly fit the central monitoring theory in terms of recognizing self as a match and non-self as a mismatch. Additionally, individual differences in these implicit and explicit measures did not correlate, suggesting that these two aspects in the sense of agency do not consist of a single process.

299 Theoretical works have proposed a distinction between implicit and explicit sense of 300 agency processing systems, owing to the presence of problematic cases of the central 301 monitoring theory in explaining the sense of agency both in healthy subjects and in 302 patients with disorders of the sense of agency (Synofzik et al., 2008a; Synofzik et al., 303 2013). It has been argued that not of all the predicted sensory signals generated from 304 our own movements will reach awareness (Castiello, Paulignan, \& Jeannerod, 1991; 305 Fourneret \& Jeannerod, 1998), and thus small discrepancies do not necessarily influence 306 the sense of agency. The importance of emotional valence (Takahata et al., 2012) and 307 beliefs as external contextual cues have also been emphasized (Synofzik et al., 2013). 308 Other studies have shown that central monitoring in patients with schizophrenia is 309 unimpaired when making predictions for the sensorimotor adjustments for grip force 310 (Delevoye-Turrell, Giersch, \& Danion, 2002), or when adjusting hand movements in 311 case of discrepancies between their own hand movements and visual consequences 312 (Fourneret, Franck, Slachevsky, \& Jeannerod, 2001; Knoblich, Stottmeister, \& Kircher, 313 2004). However, these findings do not deny the importance of sensorimotor prediction 314 and the sensory feedback in the formation of the sense of agency. Recent theories have 315 proposed an integration of various cues in two forms of agency, as an extension of the 
316 central monitoring theory (Moore \& Fletcher, 2012; Synofzik et al., 2013). Although

317 presented theoretically, only a few experiments have been conducted to support the

318 distinction of implicit and explicit sense of agency (Barlas \& Obhi, 2014; Dewey \&

319 Knoblich, 2014; Ebert \& Wegner, 2010; Moore et al., 2012).

320 This issue was approached in a single experiment by assessing the effect of 321 action-effect consistency on implicit agency and self-reported authorship (Ebert \& 322 Wegner, 2010). Action-effect consistency was defined according to whether the object 323 on the screen moved in the same, or in the opposite direction as the action. Implicit 324 agency was measured on a 10-point scale as interval estimates of how much time has 325 passed from the participants' own movement to the intended movement on the screen.

326 Explicit agency was measured on a 7-point scale in terms of how much the participants 327 felt that their movement made the object on the screen move. It has been shown that action-effect consistency affected explicit self-reported authorship more than implicit interval estimates. Additionally, implicit interval estimates and explicit authorship were correlated when asked in the same block, while they did not correlate when asked in separate blocks. This points out the problems of arbitral linkage of the interval estimates on self-reports when asked simultaneously. A study explored the association of intentional binding and explicit prediction using a dissociation paradigm of implicit and

334 explicit learning (Moore et al., 2012). In their experiment, outcomes were probabilistically caused by actions. Participants conducted the intentional binding task, and at the same time they judged the extent to which they believed there would be a tone in the next trial. The learning history of action binding showed a different pattern from that of the explicit prediction. These preceding experiments have approached the issue by introducing an explicit question into implicit agency measures. In our study we assessed the intentional binding task as implicit measure and self-other attribution task as explicit measure, and we compared the two measures when both were assessed as

342 individual tasks. The possibility of the previous question affecting the later ones was

343 avoided by assessing this in separate experiments. Our findings add the notion that the 344 two systems are separable, in line with individual differences, fitting the theoretical 345 framework as proposed by Synofzik et al. (2008a).

346 An alternative explanation that could be offered from our results is that this difference 347 is due to the different structures of the two tasks. There are ongoing discussions on the 348 backgrounds of both implicit and explicit measures. For example, there are studies 
349 suggesting that causation but not intentional action is the root of intentional binding

350 (Buehner, 2012; Dogge, Schaap, Custers, Wegner, \& Aarts, 2012). Also, the explicit

351 task has been discussed in terms of contamination by an aspect of the sense of

352 ownership of body movement instead of evaluating the sense of agency alone (Tsakiris,

353 Longo, \& Haggard, 2010). Owing to these limitations, there are possibilities that our

354 results derive from different structural backgrounds including different validity as an

355 agency task. At the same time, our results indicated that cautious interpretations would

356 be needed to evaluate the sense of agency in clinical cases by single measure.

357 Another limitation of our study is that the intentional binding effect observed in our 358 study was relatively large compared to the original study (Haggard et al., 2002).

359 However, reported amounts of binding in healthy subjects are not constant among 360 studies, and indeed there are works that report rather strong binding in healthy subjects 361 (Kranick et al., 2013; Takahata et al., 2012). Possible causes of this difference can be 362 the forms of button press as voluntary actions, or volumes and pitches of the tones as 363 feedbacks of actions, which are not being controlled among studies. The result of the 364 explicit task is also relatively different from the original study (Franck et al., 2001), 365 under-attributed in angular condition and over-attributed in delay condition. Possible 366 causes for this difference can also arise from the difference in experimental setups. 367 Compared to Franck's original study, which used a horizontal mirror to present the 368 visual feedback, we modified the apparatus and placed the computer screen directly in 369 front of the participants. The intrinsic delay of the feedback, and the time span of the 370 virtual image appearance are also different. Regardless of these differences, the 371 essentials of the evaluations have been preserved.

372 In summary, by comparing the two distinct methods of measuring the sense of agency, 373 we found supporting evidence for the dissociation of the explicit judgement of agency 374 from the lower-level experience of the feeling of agency. We suggest that a distinction 375 between these two aspects will be essential in evaluating the sense of agency in health 376 and in diseases.

\section{Acknowledgements}

379 This work was supported by Grants-in-Aid for Scientific Research A (24243061), and 380 on Innovative Areas (23120009), from the Ministry of Education, Culture, Sports, 381 Science and Technology of Japan (MEXT); Grants-in-Aid for Young Scientists A 
(23680045) from the Japan Society for the Promotion of Science - Japan (JSPS); Health and Labour Science Research Grant for Comprehensive Research on Disability Health and Welfare (H25-seishin-jitsuyouka-ippan-001) from the Ministry of Health, Labour and Welfare - Japan.

The authors wish to extend their gratitude to Takashi Tsukiura and Paeksoon Park for coordination of the study, Shikiho Dote for data acquisition, Satoshi Saito for technical support, and most of all, to the participants in the study.

\section{Conflict of interest}

391 The authors report no conflict of interest associated with this manuscript.

\section{References}

394 Barlas, Z., \& Obhi, S. S. (2014). Cultural background influences implicit but not explicit sense of agency for the production of musical tones. Consciousness and Cognition, 28, 94-103.

397 Blakemore, S. J., Wolpert, D. M., \& Frith, C. D. (2002). Abnormalities in the awareness of action. Trends in Cognitive Sciences, 6, 237-242.

399 Buehner, M. J. (2012). Understanding the past, predicting the future: causation, not 400 intentional action, is the root of temporal binding. Psychological Science, 23, 401 1490-1497.

402 Castiello, U., Paulignan, Y., \& Jeannerod, M. (1991). Temporal dissociation of motor 403 responses and subjective awareness. A study in normal subjects. Brain, 114, 2639-2655.

404 Daprati, E., Franck, N., Georgieff, N., Proust, J., Pacherie, E., Dalery, J., et al. (1997). 405 Looking for the agent: an investigation into consciousness of action and 406 self-consciousness in schizophrenic patients. Cognition, 65, 71-86.

407 David, N., Newen, A., \& Vogeley, K. (2008). The "sense of agency" and its underlying 408 cognitive and neural mechanisms. Consciousness and Cognition, 17, 523-534.

409 Delevoye-Turrell, Y., Giersch, A., \& Danion, J. M. (2002). A deficit in the adjustment of 410 grip force responses in schizophrenia. Neuroreport, 13, 1537-1539.

411 Dewey, J. A., \& Knoblich, G. (2014). Do implicit and explicit measures of the sense of 412 agency measure the same thing? PLoS One, 9, e110118.

413 Dogge, M., Schaap, M., Custers, R., Wegner, D. M., \& Aarts, H. (2012). When moving 414 without volition: implied self-causation enhances binding strength between involuntary 
actions and effects. Consciousness and Cognition, 21, 501-6.

Ebert, J. P., \& Wegner, D. M. (2010). Time warp: authorship shapes the perceived timing of actions and events. Consciousness and Cognition, 19, 481-489.

418 Farrer, C., Frey, S. H., Van Horn, J. D., Tunik, E., Turk, D., Inati, S., et al. (2008). The angular gyrus computes action awareness representations. Cerebral Cortex, 18, 254-261.

421 Fletcher, P. C., \& Frith, C. D. (2009). Perceiving is believing: a Bayesian approach to 422 explaining the positive symptoms of schizophrenia. Nature Reviews Neuroscience, 10, 423 48-58.

424 Fourneret, P., Franck, N., Slachevsky, A., \& Jeannerod, M. (2001). Self-monitoring in 425 schizophrenia revisited. Neuroreport, 12, 1203-1208.

426 Fourneret, P., \& Jeannerod, M. (1998). Limited conscious monitoring of motor 427 performance in normal subjects. Neuropsychologia, 36, 1133-1140.

428 Franck, N., Farrer, C., Georgieff, N., Marie-Cardine, M., Dalery, J., d'Amato, T., et al. (2001). Defective recognition of one's own actions in patients with schizophrenia. The American Journal of Psychiatry, 158, 454-459.

Frith, C. (2012). Explaining delusions of control: the comparator model 20 years on. Consciousness and Cognition, 21, 52-54.

Frith, C. D., Blakemore, S. J., \& Wolpert, D. M. (2000). Abnormalities in the awareness and control of action. Philosophical Transactions of the Royal Society of London. Series B, Biological Sciences, 355, 1771-1788.

Gallagher, S. (2000). Philosophical conceptions of the self: implications for cognitive science. Trends in Cognitive Sciences, 4, 14-21. Haggard, P., Clark, S., \& Kalogeras, J. (2002). Voluntary action and cofnscious awareness. Nature Neuroscience, 5, 382-385.

441 Awareness of action in schizophrenia. Neuroreport, 14, 1081-1085.

442 Knoblich, G., Stottmeister, F., \& Kircher, T. (2004). Self-monitoring in patients with 443 schizophrenia. Psychological Medicine, 34, 1561-1569.

444 Kranick, S. M., Moore, J. W., Yusuf, N., Martinez, V. T., LaFaver, K., Edwards, M. J., et 445 al. (2013). Action-effect binding is decreased in motor conversion disorder: implications 446 for sense of agency. Movement Disorders, 28, 1110-1116.

447 Libet, B., Gleason, C. A., Wright, E. W., \& Pearl, D. K. (1983). Time of conscious 
448 intention to act in relation to onset of cerebral activity (readiness-potential). The 449 unconscious initiation of a freely voluntary act. Brain, 106, 623-642.

450 Maeda, T., Kato, M., Muramatsu, T., Iwashita, S., Mimura, M., \& Kashima, H. (2012). 451 Aberrant sense of agency in patients with schizophrenia: forward and backward 452 over-attribution of temporal causality during intentional action. Psychiatry Research, 453 198, 1-6.

454 Maeda, T., Takahata, K., Muramatsu, T., Okimura, T., Koreki, A., Iwashita, S., et al. 455 (2013). Reduced sense of agency in chronic schizophrenia with predominant negative 456 symptoms. Psychiatry Research, 209, 386-392.

457 Moore, J., \& Haggard, P. (2008). Awareness of action: Inference and prediction. 458 Consciousness and Cognition, 17, 136-144.

459 Moore, J. W., \& Fletcher, P. C. (2012). Sense of agency in health and disease: a review 460 of cue integration approaches. Consciousness and Cognition, 21, 59-68.

461 Moore, J. W., Middleton, D., Haggard, P., \& Fletcher, P. C. (2012). Exploring implicit 462 and explicit aspects of sense of agency. Consciousness and Cognition, 21, 1748-1753.

463 Moore, J. W., Wegner, D. M., \& Haggard, P. (2009). Modulating the sense of agency 464 with external cues. Consciousness and Cognition, 18, 1056-1064.

465 Nielsen, T. I. (1963). Volition: a new experimental approach. Scandinavian Journal of 466 Psychology, 4, 225-230.

467 Oldfield, R. C. (1971). The assessment and analysis of handedness: the Edinburgh 468 inventory. Neuropsychologia, 9, 97-113.

469 Synofzik, M., Vosgerau, G., \& Newen, A. (2008a). Beyond the comparator model: a 470 multifactorial two-step account of agency. Consciousness and Cognition, 17, 219-239.

471 Synofzik, M., Vosgerau, G., \& Newen, A. (2008b). I move, therefore I am: a new 472 theoretical framework to investigate agency and ownership. Consciousness and 473 Cognition, 17, 411-424.

474 Synofzik, M., Vosgerau, G., \& Voss, M. (2013). The experience of agency: an interplay 475 between prediction and postdiction. Frontiers in Psychology, 4, 127.

476 Takahata, K., Takahashi, H., Maeda, T., Umeda, S., Suhara, T., Mimura, M., et al. 477 (2012). It's not my fault: postdictive modulation of intentional binding by monetary 478 gains and losses. PLoS One, 7, e53421.

479 Tsakiris, M., Longo, M. R., \& Haggard, P. (2010). Having a body versus moving your 480 body: neural signatures of agency and body-ownership. Neuropsychologia, 48, 
$481 \quad 2740-2749$.

482 Voss, M., Moore, J., Hauser, M., Gallinat, J., Heinz, A., \& Haggard, P. (2010). Altered 483 awareness of action in schizophrenia: a specific deficit in predicting action 484 consequences. Brain, 133, 3104-3112.

485 Wegner, D. M. (2003). The mind's best trick: how we experience conscious will. Trends 486 in Cognitive Sciences, 7, 65-69.

487 Wolpe, N., Moore, J. W., Rae, C. L., Rittman, T., Altena, E., Haggard, P., et al. (2014). 488 The medial frontal-prefrontal network for altered awareness and control of action in 489 corticobasal syndrome. Brain, 137, 208-220.

490 


\section{$491 \quad$ Figure captions}

492 Figure 1

493 Perceived times of actions and tones in experiment 1. Actions were perceived as shifted

494 toward their subsequent tones, while tones were perceived as shifted towards the 495 preceding action that caused them.

496

497 Figure 2

498 Number of "Yes" responses when participants were asked whether movements on the

499 screen corresponded to their own computer movements in experiment 2. (A) with 500 angular bias, and (B) with temporal bias

501

502 
Figure 1

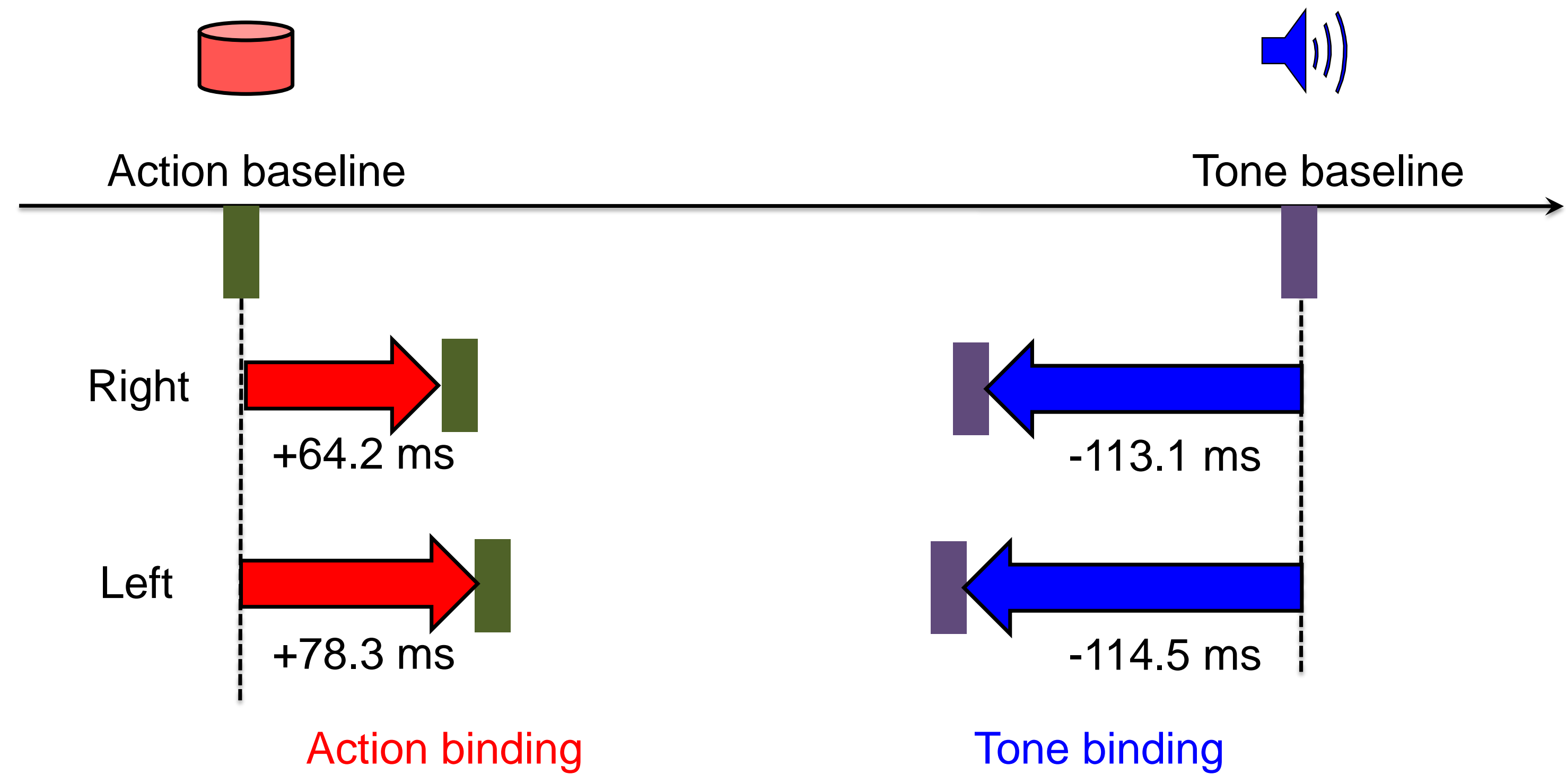


Figure 2

\section{(A)}

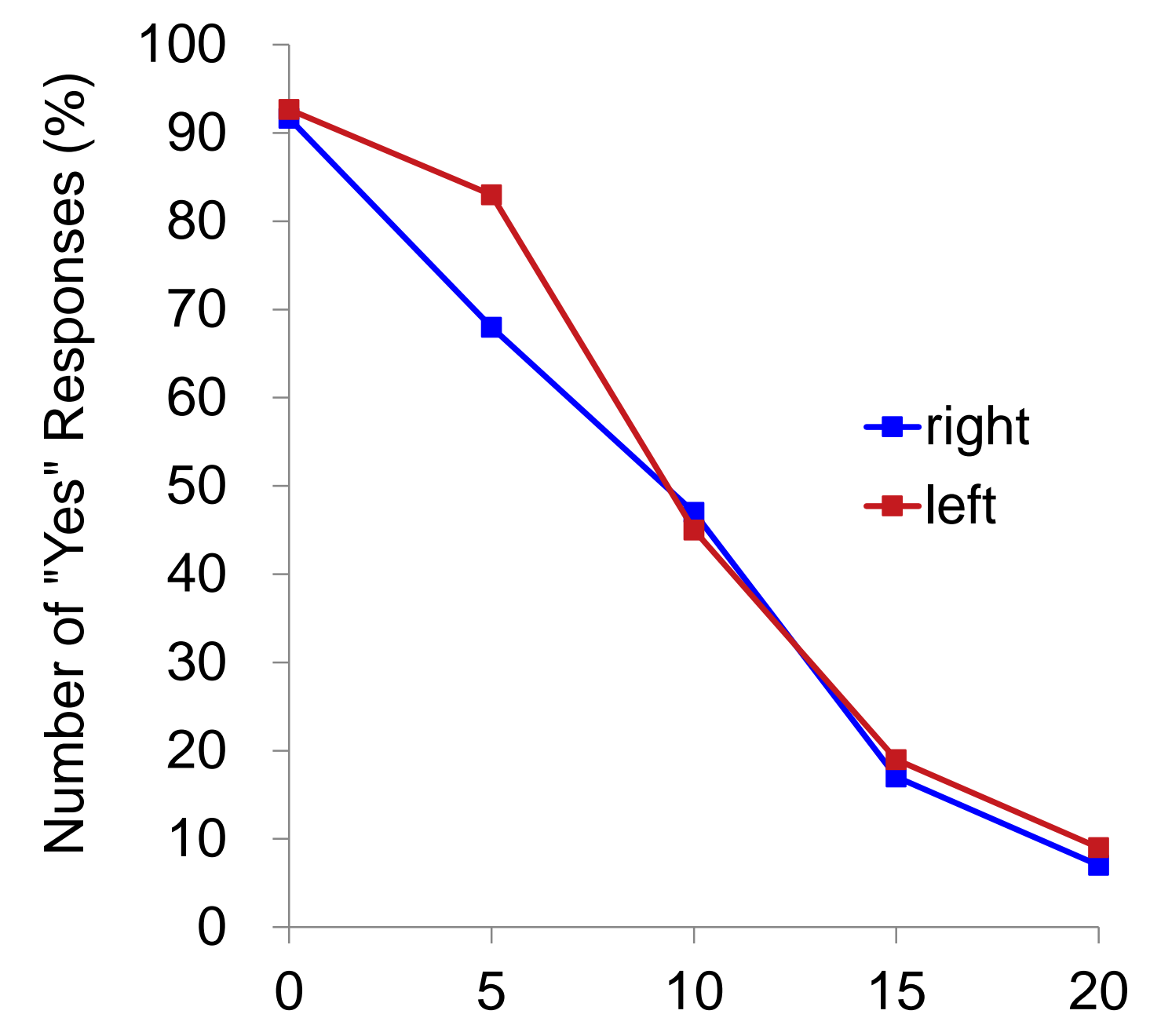

Angular bias (degrees)

\section{(B)}

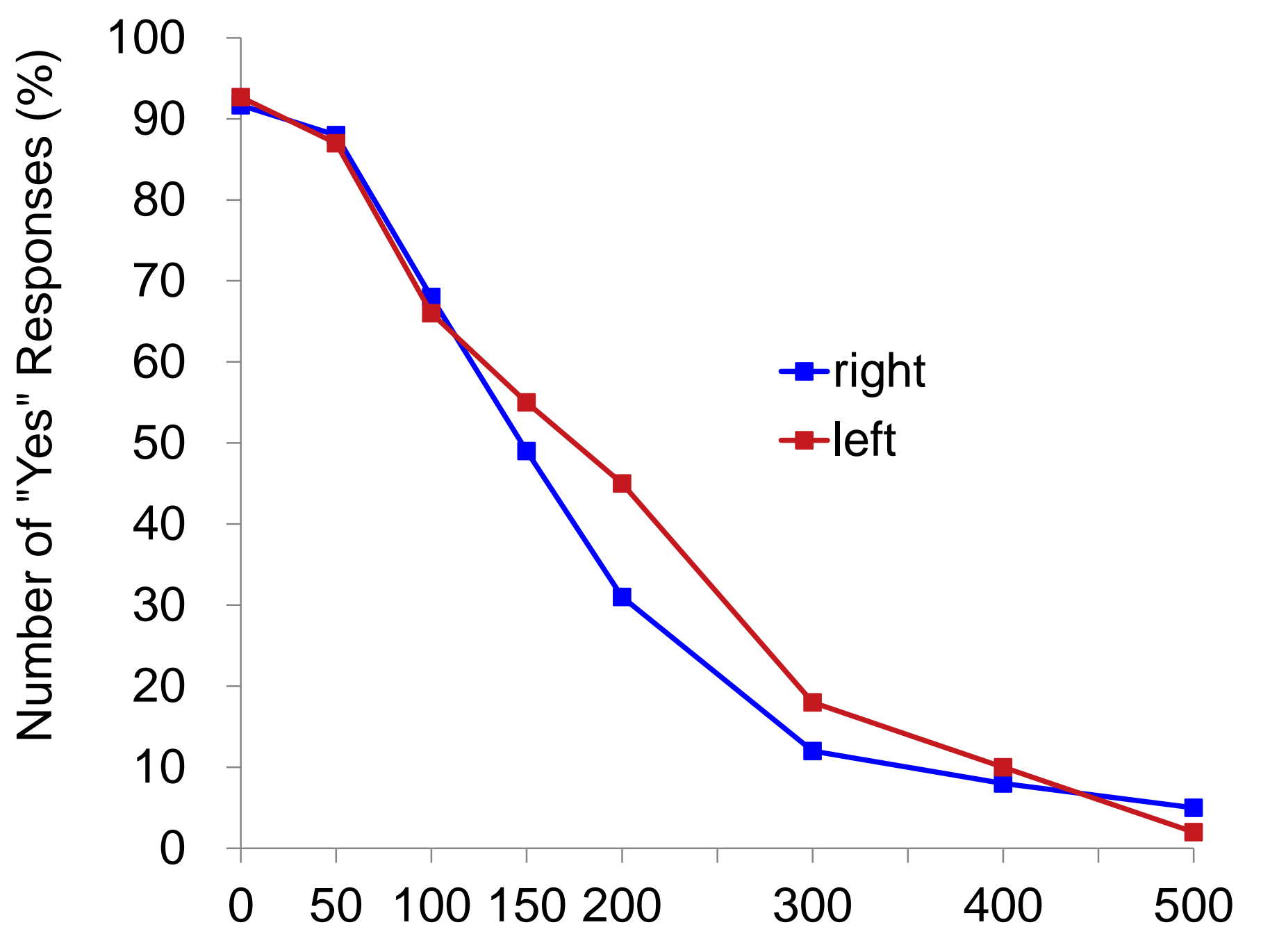

Temporal bias (ms) 
503 Table 1

504 Correlations between scores of implicit task and explicit task

\begin{tabular}{lccc}
\hline & \multicolumn{3}{c}{ Implicit task } \\
\cline { 2 - 4 } & Action binding & Tone binding & Overall binding \\
\hline Explicit task & & & \\
Slope coefficient & & & 0.013 \\
$\quad$ Angular biases & 0.217 & 0.196 & 0.174 \\
Temporal biases & 0.064 & -0.139 & \\
& & & -0.089 \\
Number of yes responses & & & 0.021 \\
$5^{\circ}$ angular bias & 0.039 & 0.128 & 0.181 \\
$10^{\circ}$ angular bias & 0.201 & 0.120 & 0.200 \\
$150 m$ m temporal bias & -0.032 & -0.315 & \\
$200 m s$ temporal bias & 0.003 & -0.281 & \\
\hline
\end{tabular}

Spearman rank correlations. None showed significant $(p<0.05)$ correlations.

505 